\title{
Acute airway obstruction - an unusual cause
}

\author{
D Williamson, T Llewellyn
}

A 69 year old woman arrived in the emergency department after a collapse at home. The ambulance crew found her in a low cardiac output state, unresponsive other than to painful stimulation. On arrival she was bradycardic, hypotensive, and deeply cyanosed. Ventilation by means of a bag-and-mask system could not be achieved; she was therefore intubated. Her condition improved after atropine $0.6 \mathrm{mg}$ and adrenaline $1 \mathrm{mg}$ as well as $100 \%$ oxygen. Ventilation remained very difficult due to high inflation pressures. This remained the case despite intravenous salbutamol, frusemide, paralysis, and a change of endotracheal tube. Radiography (fig 1) suggested a large right sided mediastinal mass. Subsequent thoracic computed tomography (fig 2) confirmed the presence of a mass that appeared aneurysmal causing compression of the trachea at the level of the carina. She also had a midshaft fracture of her right femur, which appeared pathological. A history of recent weight loss, dyspepsia, and onset of atrial fibrillation together with an essentially normal chest radiograph two months before presentation led to the presumptive diagnoses of a mitotic aneurysm of the thoracic aorta. Given the poor prognoses and her premorbid dependence it was felt that conservative management was most appropriate. Despite regaining consciousness for a short time she gradually deteriorated over a 24 hour period and died peacefully on the intensive therapy unit. The postmortem examination confirmed the presence of a haemorrhagic mass in the anterior mediastinum. Histology identified the tumour as an infiltrating leiomyosarcoma involving both atria and the pericardium, completely surrounding the carina and compressing the superior vena cava.

It would appear that an acute bleed into a pre-existing mediastinal tumour caused the acute airway obstruction. Although this was suspected from the outset, careful elimination of more common causes must not be neglected. Foreign body inhalation, broncho-

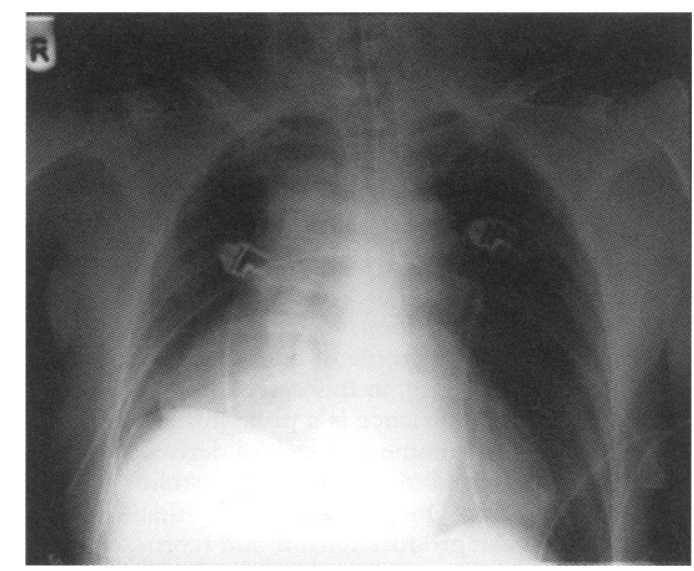

Figure 1 Chest radiograph.

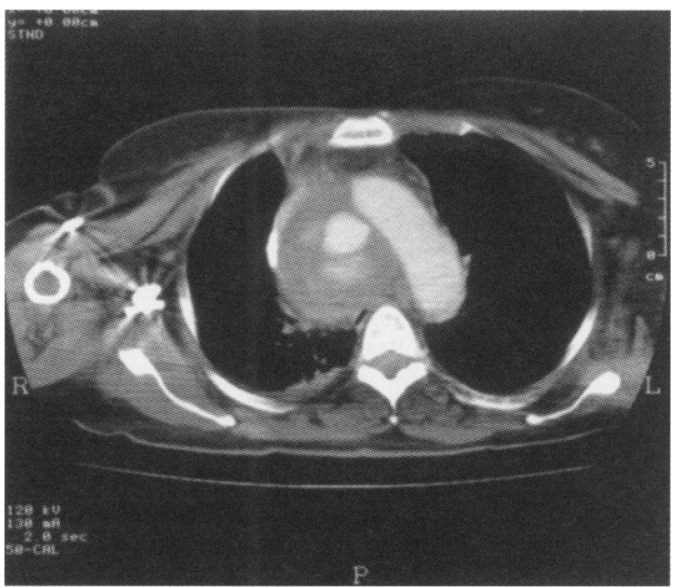

Figure 2 Computed tomogram.

spasm, and laryngospasm were all considerations in this case, as were a blocked or kinked endotracheal tube, poor positioning, and cuff herniation after intubation. Additional measures reported to have helped in acute extrinsic tracheal compression are nebulised adrenaline ${ }^{1}$ and mechanical relief with direct laryngoscopy. ${ }^{2}$

1 Dearlove OR, Barrie J. Use of nebulised adrenaline in tracheal compression. Br f Hosp Med 1995;53:179.

2 deSoto $H$. Direct laryngoscopy as an aid to relieve airway obstruction in a patient with a mediastinal mass. Anesthesiology 1987;67:116-17. 